\title{
Lo que es puede no ser lo que parece: A propósito de las altas capacidades
}

\author{
Sílvia Mayoral-Rodríguez ${ }^{1}$, Carme Timoneda-Gallart ${ }^{1}$ y \\ Frederic Pérez-Álvarez ${ }^{2}$ \\ ${ }^{1}$ Universidad de Girona (España); \\ ${ }^{2}$ Hospital Universitario Dr.Trueta de Girona (España)
}

\begin{abstract}
Se estudió el DN-CAS versus el WISC para altas capacidades con aprendizaje o conductas disfuncionales (doble excepcionalidad). 114 casos de alta capacidad, entre 10 y 14 años (56\% mujeres), 54 con dificultades de aprendizaje y 60 con conductas disfuncionales, fueron evaluados con el DN-CAS (PASS) y el WISC. Un análisis factorial mostró cuatro factores PASS y uno WISC. El WISC cargó principalmente en el simultáneo. Ningún otro procesamiento PASS cargó en el WISC. Se halló débil o moderada correlación entre DN-CAS y WISC. El simultáneo es el proceso menos correlacionado por lo que puede inferirse es el más independiente. El análisis estadístico descriptivo mostró que el simultáneo fue el procesamiento PASS con mayor puntuación y el procesamiento atención fue el con menor puntuación. En el análisis de agrupamiento de los procesos PASS superiores a 115 (+1DS) y 130 (+2DS), el simultaneo es el más frecuentemente involucrado. Por último, un patrón singular (patrón "N") aparece como marcador de disfunción emocional ligada al planning. En resumen, se demuestra la estructura de cuatro factores del DNCAS. El simultáneo aparece ligado al WISC. Un buen o excelente simultáneo aparece en alta capacidad. El DN-CAS es ventajoso para alta capacidad con aprendizaje o conducta disfuncional (doble excepcionalidad) pues el DN-CAS valora planificación, creatividad y metacognición.
\end{abstract}

Palabras clave: Alta capacidad, problema aprendizaje, disfunción conductual, doble excepcionalidad, PASS.

What it is may not be what it seems. About the high capacities. This study examines the DN-CAS versus WISC to assess gifted children with learning difficulties or conduct disorders (twiceexceptional children). 114 gifted children, from 10 years to 14 years old (female 56\%), 54 with learning difficulties, 60 with conduct disorder, were assessed on DN-CAS (PASS) and WISC. Factor analysis showed four PASS factors and one WISC factor. WISC loaded mainly on simultaneous. No other PASS processing was linked with WISC. Correlations showed weak or moderate correlation between CAS and WISC. Simultaneous is the less correlated process and therefore we can infer that it woks more independently. Descriptive statistics showed that simultaneous was the higher score and planning the lower score. On analyzing clusters of PASS processes higher that $115(+1 \mathrm{SD})$ and $130(+2 \mathrm{SD})$, simultaneous appears as the most frequently involved. Finally, a particular pattern ("N" pattern) is found as a marker of emotional dysfunction linked to planning. These findings demonstrate a four-factor solution for D-N CAS among the gifted children tested. Only simultaneous appears linked to WISC. A good or excellent simultaneous appears linked to gifted children. The DN-CAS seems to offer an advantageous tool to assess gifted children, mainly in the case of underachievers or disturbed behavior, given that DN-CAS assesses planning, closely related to creativity and metacognition.

Keywords: Giftedness, underachievers, behavioral dysfunction, twice-exceptional, PASS.

Correspondencia: Silvia Mayoral Rodríguez. Universidad de Girona. C/ Pujada de Sant Domènec, $\mathrm{n}^{\circ}$ 9. C.P.: 17004. Girona (España). E-mail: silvia.mayoral@udg.edu 
La definición de alta capacidad está sujeta a cierta variabilidad, pero un criterio común es que debe sustentarse en más que la sola alta capacidad definida por tests psicométricos, aunque, a efectos operativos, se contempla un CI de al menos 130 (98 ${ }^{\text {th }}$ percentil) (Alberta Education, 2012, p.6) (U.S. Department of Education, 1995). Actualmente, se acepta que la alta capacidad requiere la excelencia en determinadas áreas como la creatividad y liderazgo, por ejemplo (Sternberg, Jarvin, y Grigorenko, 2011).

Los resultados de los estudios en altas capacidades son complejos. Queremos resaltar ejemplos de aquellos que tratan aspectos en que nos centraremos, particularmente, planificación y metacognición. Calero, García-Martín, y Robles (2011) encontraron una mejor auto-regulación. Se ha publicado una mayor eficiencia en resolver problemas complejos (Sternberg, Jarvin, y Grigorenko, 2011) con la utilización de mejores estrategias y un mejor desempeño en metacognición y auto-regulación (Shore, 2000; Steiner, 2006). Particularmente, se ha publicado una mejor función ejecutiva y planificación (estrategias) en situaciones de aprendizaje (Shore, 2000). Una mejor metacognición ha sido publicada en alta capacidad en edad preescolar y, también, en edad escolar y adolescencia cuando son capaces de usar estrategias metacognitivas similares a las de los adultos (Shore, 2000; Steiner y Carr, 2003).

Paradójicamente, sin embargo, se han publicado dificultades metacognitivas asociadas a dificultades de aprendizaje (Nicpon, Allmon, Sieck, y Stinson, 2010) en altas capacidades. Por ejemplo, un estudio comprobó gran variabilidad en la resolución de pruebas de función ejecutiva (Hernández, Speirs, Burney, y Cook, 2014). El corolario de los estudios sobre el tema es que estudiantes con altas capacidades no siempre obtienen brillantes resultados académicos. Llamativamente, se ha podido constatar una no despreciable incidencia de dificultades de aprendizaje en este colectivo (Reis y Coach, 2000; Robinson, 1999). Del mismo modo, conductas disfuncionales se asocian, no infrecuentemente, a este colectivo. Así, problemas atencionales, falta de motivación, falta de actitud en tareas irrelevantes, enfrentamiento con la autoridad, cuestionamiento de las normas, hipercinesia, problemas de sueño (Craggs, Sanchez, Kibby, Gilger, y Hynd, 2006; Dai, 2005; Dole, 2000; Webb, 2001; Weinfeld, Barnes-Robinson, Jeweler, y Shevitz, 2002). Por cierto, algunas de estas conductas son características del TDAH.

Hasta donde sabemos, solo dos estudios han examinado el PASS (Planificación, Atención, Sucuencial, Simultáneo) en altas capacidades (Schofield y Ashman, 1987; Nagleri y Das, 1997). Schofield and Ashman encontraron que la alta capacidad se asociaba a mejor planificación y simultaneo, con la limitación de que los investigadores no tomaron medidas rigurosas para asegurar que se habían utilizado los mismos criterios para definir la alta capacidad. Por su parte, Naglieri y Das hallaron que la alta capacidad se asociaba a mejor simultaneo y secuencial, pero con la limitación de no haber seleccionado la muestra con un procedimiento riguroso. 
En este estudio establecimos la hipótesis de que podríamos replicar la estructura del DN-CAS en cuatro factores. También, que el DN-CAS, sin pruebas significativamente influenciables por el conocimiento adquirido como es el caso de, por ejemplo, Aritmética, Información y Vocabulario del Wechsler, sería más fiable y consistente. Asimismo, establecimos la hipótesis de que la alta capacidad asociada a problemas de aprendizaje o/y conductas disfuncionales tendría relación con la planificación. Finalmente, sobre la base de nuestra amplísima experiencia con el DN-CAS establecimos que un perfil particular, el perfil "N", estaría presente asociado a los casos con problemas de aprendizaje o/y conductas disfuncionales como marcador o indicador de una planificación disfuncional asociada a una disfunción emocional.

\section{MÉTODO}

\section{Participantes}

Un total de 114 sujetos de entre 10 y 14 años de edad (56\% de mujeres) constituyeron la muestra. Se considera que un diagnóstico de alta capacidad es suficientemente fiable a estas edades según la maduración cognitiva. Todos $(n=114)$ cumplían criterios de alta capacidad. El diagnóstico se basó en habilidades verbales, numéricas y razonamiento lógico y especial según la batería BAdyG (Yuste, Martínez, y Galve, 2002). Se valoró la creatividad según PIC-N (Artola, Ancillo, Barraca, y Mosteiro, 2010), PIC-J (Artola et al., 2008) y CREA (Corbalán et al., 2003). La comprensión verbal, el razonamiento perceptual, la memoria de trabajo y la velocidad de procesamiento se valoraron según Wechsler (2003).

Todos los miembros de la muestra tenían un CI de 130 o superior según Wechsler. 54 sujetos cumplían criterios DSM-5 $(\mathrm{A}+\mathrm{B}+\mathrm{C}+\mathrm{D})$ de "specific learning disability (SLD)".60 sujetos cumplían criterios DSM-5 de trastorno de conducta. Cabe recordar que conductas de agresión encubierta como mentir, ignorar, hacer el vacío y extender rumores son más propias de las mujeres, en tanto que conductas como la violencia física y la impulsividad son más propias de los hombres. Un grupo control, comparable en edades y sexo, fue seleccionado de una base de datos conteniendo 1987 sujetos atendidos en nuestro centro.

Los datos sobre aprovechamiento académico fueron informados por el profesorado. Se considera que el profesorado es una fuente de información fiable en este tipo de estudios. La persona investigadora usó una escala ordinal Likert (de 1 a 7 , desde "muy mal" a "muy bien") para graduar la relevancia del problema de aprendizaje y la disfunción conductual. El personal investigador tenía no menos de 5 años de experiencia "postgrado" en una amplia variedad de casos relativos a problemas de aprendizaje y disfunciones conductuales. 
Un cribado medico fue llevado a cabo para descartar enfermedad, particularmente, en las áreas de salud neurológica y mental. Según necesidad, se realizaron los siguientes estudios: exploración oftalmológica y otorrinolaringológica, EEG, potenciales evocados auditivos y visuales, polisomnografía, y otros. Se investigó la toma de medicación. La presencia de enfermedad o toma de medicación fueron criterios de exclusión.

\section{Instrumentos}

Se administró a todos los sujetos $(n=114)$ la batería DNCAS (Sistema de Evaluación Cognitiva de Das y Naglieri) traducida y validada para su aplicación en catalán (Pérez y Timoneda, 2007). Esta batería evalúa el procesamiento PASS, a saber, Planificación, Atención, Sucesivas y Simultáneas. Las pruebas de planificación son: números Apareando números, códigos establecidos y conexiones. Los de atención son: atención expresiva, detección de números y atención receptiva. Las pruebas simultáneas son: matrices no verbales, relaciones verbal-espaciales y memoria de figuras. Los sucesivos son: series de palabras, repetición de frases, frase y pregunta (de 8 a 17 años) y velocidad del habla (de 5 a 7 años). Cada una de las cuatro escalas PASS rinde una puntuación estándar con una media normativa de 100 y una desviación estándar $(S D)$ de 15. Para las tres subpruebas de cada una de las cuatro escalas, la media es 10 y la $S D$ es 3 . Apareando números requiere que los niños diseñen una estrategia para encontrar y subrayar dos números que son iguales en una fila. Los números aumentan en longitud de un dígito a siete dígitos. Los códigos establecidos muestran un conjunto distinto de códigos y disposiciones de filas y columnas. Una leyenda en la parte superior de cada página muestra cómo las letras corresponden a códigos simples (por ejemplo, A, B, C, D corresponden a OX, XX, OO, $\mathrm{XO}$, respectivamente).

Los niños deben completar los códigos apropiados en casillas vacías debajo de cada letra buscando la manera más eficiente posible (plan). Las conexiones requieren que los niños conecten de manera correcta números en secuencia o números y letras en órdenes alternas. La atención expresiva exige que los niños nombren el color de la tinta en las palabras azul, amarillo, verde y rojo que se imprimen según el fenómeno de Stroop. La detección de números consiste en páginas de números en diferentes formatos. Los niños deben encontrar, por ejemplo, los números 1, 2 y 3 en una página que contiene muchos distractores (por ejemplo, el mismo número impreso en diferentes fuentes). Se tiene en cuenta la precisión (correcto menos detecciones falsas). La atención receptiva exige que el niño identifique pares de letras que cumplan criterios específicos entre muchos pares de letras que no lo hacen. Las matrices no verbales muestran formas y diseños geométricos que están interrelacionados a través de la organización espacial o lógica. Las relaciones verbal-espaciales muestran dibujos y una pregunta impresa; por ejemplo, "¿qué imagen muestra un círculo a la izquierda de una cruz debajo de un triángulo sobre un cuadrado?" 
La memoria de figuras requiere que el niño identifique un diseño geométrico cuando está incrustado en una figura compleja. La serie de palabras exige que el niño repita las palabras en el mismo orden que el examinador. La repetición de frases requiere que el niño repita oraciones, como "el azul se pone amarillo", que el examinador lee en voz alta. Las preguntas sobre frases (para la edad de entre 8 y 17 años) usan las mismas oraciones anteriores, pero de una manera diferente. Los niños escuchan una frase y luego se les hace una pregunta sobre ella. Por ejemplo, la oración: "El azul se está poniendo amarillo". La pregunta: "¿quién está amarillento?" La respuesta: "el azul". La velocidad del habla requiere que el niño repita una serie de palabras en un orden lineal particular. Por otro lado, la medida de cociente intelectual se obtuvo con la administración del WISC-IV a cada sujeto (Wechsler, 2003).

\section{Procedimiento}

Los participantes pasaron los tests individualmente en una sala tranquila habilitada a tal efecto. Cada test requirió aproximadamente una hora y media de tiempo, empleando minutos de descanso entre las diferentes subescalas. El orden de administración fue constante. Las puntuaciones se obtuvieron de acuerdo al manual correspondiente (para el D-N CAS, Naglieri y Das, 1997). Se solicitó consentimiento informado a padres o responsables de su custodia. El estudio fue aprobado por el comité ético de nuestra institución.

\section{RESULTADOS}

Realizamos un análisis de Componentes Principales con rotación (Varimax) para examinar la estructura factorial del DN-CAS y WISC.

Conforme a nuestra hipótesis, la estructura factorial hallada (Tabla 1) indicó que el DN-CAS y WISC representan cinco factores con "eigenvalue" mayor de 1, explicando el 66\% de la varianza. Factor 1, Planificación. Factor 2, Secuencial. Factor 3, Simultaneo. Factor 4, Atención.

Factor 5, WISC. El "eigenvalue" fue, en orden descendente, 7.00, 2.50, 1.49, 1.25 , y 0.98 , explicando $43.75 \%, 15.68 \%, 9.35 \%, 6.11$, y $4.94 \%$ de la varianza respectivamente. Los cinco factores explicaban el $80 \%$ de la varianza. Todos los "ítems" cargaban significativamente en el factor correspondiente con valores entre 0.67 y 0.95 . 
MAYORAL-RODRÍGUEZ et al. Alta capacidad con doble excepcionalidad

Tabla 1. Análisis Componentes Principales. WISC y DN-CAS ( $n=114)$

\begin{tabular}{|c|c|c|c|c|c|}
\hline & PLAN & $\mathrm{AT}$ & SE & SIM & WISC \\
\hline \multicolumn{6}{|c|}{ DN-CAS } \\
\hline Planificación & .812 & .128 & .256 & .114 & .103 \\
\hline Emparejamiento números & .886 & .345 & .307 & -.011 & .129 \\
\hline Planificación conexiones & .642 & .247 & .164 & .238 & .072 \\
\hline Atención & .021 & .879 & .191 & .154 & .294 \\
\hline Atención expresiva & .211 & .747 & .409 & -.010 & .304 \\
\hline Detección de números & .132 & .730 & -.123 & .305 & .187 \\
\hline Secuencial & .245 & .253 & .895 & .149 & .243 \\
\hline Serie de palabras & .346 & .306 & .866 & .134 & .148 \\
\hline Repetición de frases & .131 & .128 & .808 & .126 & .307 \\
\hline Simultáneo & .098 & .148 & .131 & .917 & .063 \\
\hline Matrices No-Verbales & .046 & .037 & .126 & .848 & .079 \\
\hline Memoria de figuras & .212 & .174 & .024 & .730 & .021 \\
\hline \multicolumn{6}{|c|}{ WISC } \\
\hline Verbales & .267 & .270 & .117 & .258 & .908 \\
\hline Información & -.015 & -.001 & .082 & .153 & .836 \\
\hline Semejanzas & .212 & .220 & .200 & .083 & .819 \\
\hline Vocabulario & .227 & .247 & .205 & .067 & .833 \\
\hline Aritmética & .236 & .369 & -.220 & .515 & .437 \\
\hline Comprensión & .286 & .268 & .205 & .183 & .584 \\
\hline Dígitos & .335 & .388 & .639 & .088 & .212 \\
\hline Manipulativas & .368 & .359 & .284 & .741 & .347 \\
\hline Figuras incompletas & -.035 & -.028 & .329 & .187 & .668 \\
\hline Historietas & .039 & .030 & .162 & .701 & .228 \\
\hline Cubos & .327 & .300 & .350 & .554 & .170 \\
\hline Rompecabezas & .118 & .090 & -.077 & .852 & .191 \\
\hline Claves & .401 & .763 & .313 & .311 & .065 \\
\hline Laberintos & .247 & .489 & .334 & .497 & .146 \\
\hline Porcentaje de la varianza & 43.75 & 15.68 & 9.35 & 6.11 & 4.94 \\
\hline
\end{tabular}

Nota. Análisis Componentes Principales, Rotación Ortogonal. Aparecen resaltados los valores más significativos. PLAN es planificación. AT es atención. SE es secuencial. SIM es simultáneo

Como análisis complementario, se buscó el coeficiente de correlación (Pearson) (Tabla 2). Se aplicaron los criterios de Cohen, es decir, débil: $r \geq 0.10$, moderada: $r \geq 0.30, \mathrm{y}$ alta: $r \geq 0.50$. Se observa una alta correlación $(r \geq 0.50)$ entre los tests WISC (rs entre 0.71 y 0.92). Por contra, la correlación entre WISC y DN-CAS resultó ser débil o moderada. 
MAYORAL-RODRÍGUEZ et al. Alta capacidad con doble excepcionalidad

Tabla 2. Correlación entre subescalas del WISC y DN-CAS

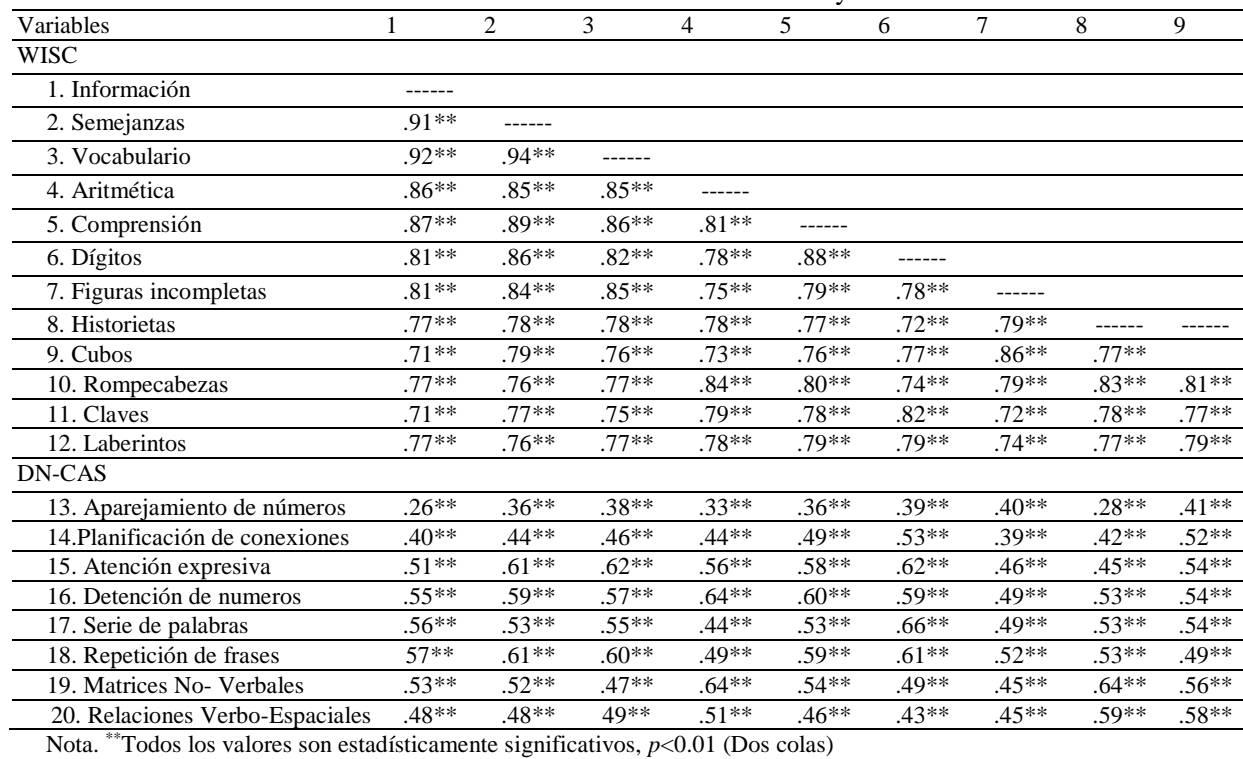

Tabla 2(continuación). Correlación entre subescalas del WISC y DN-CAS

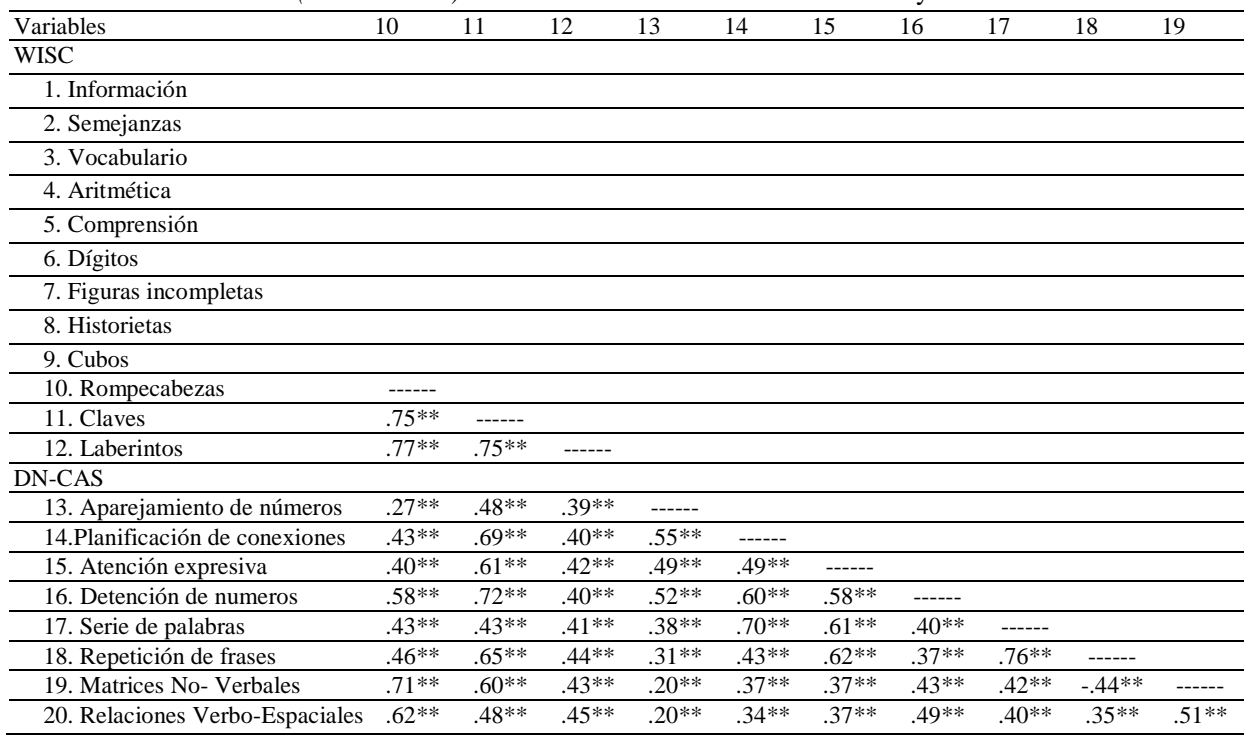

Nota. ${ }^{* *}$ Todos los valores son estadísticamente significativos, $p<0.01$ (Dos colas)

De la correlación dentro del DN-CAS cabe destacar que planificación y atención expresan entre sí una alta positive correlación (rs entre 0.49 y 0.60). En cambio, el 
simultáneo, aunque correlaciona con planificación, atención y secuencial (rs entre 0.20 y 0.49 ), es el procesamiento menos correlacionado, indicando que funciona más independientemente con respecto al resto de procesamientos.

La tablas 3 y 4 presentan estadística descriptiva de resultados del DN-CAS en la muestra de alta capacidad. El procesamiento PASS con mejor puntuación media es el simultáneo, 122.74, claramente por encima de +1 DS. El segundo mejor es la atención, 115.34, esto es, +1 SD. Luego, el secuencial, 111.20, muy próximo a la media normalizada. El peor resultado, aunque muy próximo al anterior, lo arroja la planificación, 109.70. Completamos el análisis con un escrutinio de la distribución de los procesos PASS, según resultados del DN-CAS >115 y >130 (Tablas 5 y 6). Investigamos el número de casos con uno, dos, tres y cuatro procesamientos $>115 \mathrm{y}>130$.

Tabla 3. DN-CAS. Estadística descriptiva (Tests)

\begin{tabular}{lccc}
\hline Variable & $N$ & Media & $S D$ \\
\hline & 114 & 135.58 & 4.53 \\
\hline Planificación & 114 & 109.79 & 11.73 \\
\hline Simultáneo & 114 & 122.74 & 9.61 \\
\hline Atención & 114 & 115.34 & 10.42 \\
\hline Secuencial & 114 & 111.20 & 6.19 \\
\hline Nota. Puntuación típica: 100 (media), 115 (+1DS), 130 (+2DS)
\end{tabular}

Tabla 4. DN-CAS. Estadística descriptiva (Sub-Tests)

\begin{tabular}{lllll}
\hline Variable & Sub-test & $N$ & Media & \multicolumn{1}{c}{$S D$} \\
\hline \multirow{3}{*}{ Planificación } & Aparejamiento de Números & 114 & 11.61 & 2.67 \\
\cline { 2 - 5 } & Planificación de Códigos & 114 & 11.05 & 1.90 \\
\cline { 2 - 5 } & Planificación de Conexiones & 114 & 12.08 & 2.59 \\
\hline \multirow{3}{*}{ Simultáneo } & Matrices No verbales & 114 & 13.92 & 1.76 \\
\cline { 2 - 5 } & Relaciones Verbo-Espaciales & 114 & 12.37 & 2.64 \\
\cline { 2 - 5 } Atención & Memoria de Figuras & 114 & 14.79 & 2.12 \\
\hline \multirow{3}{*}{ Secuencial } & Atención Expresiva & 114 & 12.63 & 1.79 \\
\cline { 2 - 5 } & Detección de Números & 114 & 12.47 & 1.92 \\
\cline { 2 - 5 } & Atención receptiva & 114 & 12.26 & 2.15 \\
\cline { 2 - 5 } & Serie de Palabras & 114 & 10.71 & 1.52 \\
\cline { 2 - 5 } & Repetición de Frases & 114 & 11.82 & 1.45 \\
\cline { 2 - 5 } & Frase y pregunta & 114 & 13.32 & 1.47 \\
\hline
\end{tabular}

Nota. Puntuación típica: 10 (media), 13 (+1DS), 16 (+2DS)

La tabla 5 muestra resultados de procesamientos PASS $>115$. En el $70.8 \%$ $(2.6+21.0+10.5+2.6+34.1=70.8)$ del $89.3 \%$ de casos con algún proceso $>115$ el procesamiento implicado es el simultaneo $>115$. En el $47.5 \%$ $(2.6+21.0+5.3+2.6+10.5+5.3=47.3)$ del $89.3 \%$ de casos con algún proceso $>115$ el procesamiento implicado es la atención $>115$. El secuencial obtiene peores resultados y la planificación los obtiene todavía peores. 
Tabla 5. Distribución según DN-CAS PASS >115 $(N=114)$

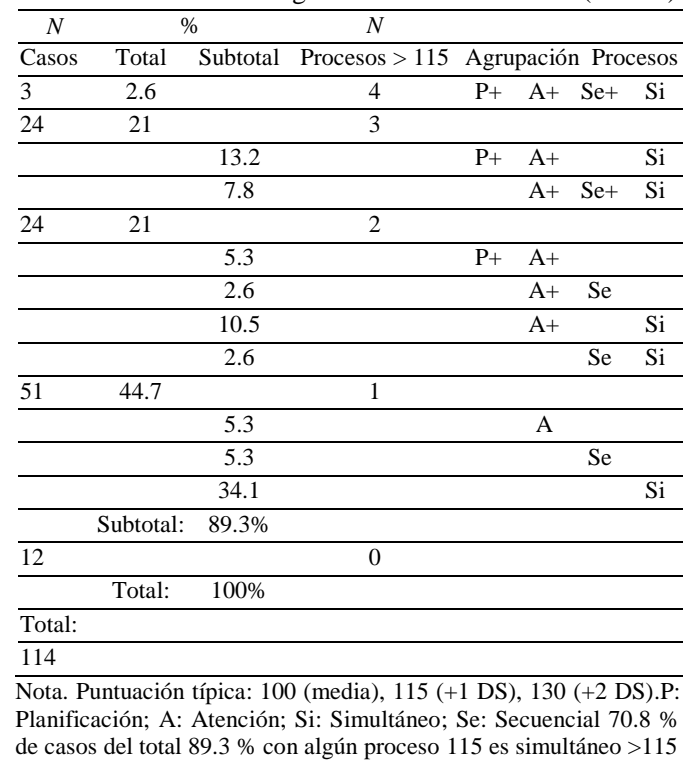

La tabla 6 muestra resultados de procesamientos PASS $>130$. De nuevo, el simultáneo es el mejor. En el 23.6\% (2.6+2.6+18.4=23.6) del 28.8\% de casos con algún procesamiento $>130$, el procesamiento implicado es el simultaneo $>130$. Solamente en el $7.8 \%(2.6+2.6+2.6=7.8)$ del $28.8 \%$ de casos con algún procesamiento $>130$ el procesamiento implicado es la planificación $>130$. Menores porcentajes corresponden a atención, $5.2 \%(2.6+2.6)$ y a secuencial, $0.0 \%$.

Tabla 6. Distribución según DN-CAS PASS>130 $(N=114)$

\begin{tabular}{|c|c|c|c|c|c|}
\hline$N$ & & $\%$ & $N$ & \multirow{2}{*}{ Agrupación } & \multirow{2}{*}{ Procesos } \\
\hline$\overline{\text { Casos }}$ & Total & Subtotal & Procesos $>130$ & & \\
\hline 0 & 0 & & 4 & & \\
\hline 3 & 2.6 & & 3 & $\mathrm{~A}+$ & $\mathrm{Si}$ \\
\hline 3 & 2.6 & & 2 & $\mathrm{P}+$ & $\mathrm{Si}$ \\
\hline \multirow[t]{5}{*}{27} & 23.6 & & 1 & & \\
\hline & & 2.6 & & $\mathrm{P}$ & \\
\hline & & 2.6 & & $\mathrm{~A}$ & \\
\hline & & 18.4 & & & $\mathrm{Si}$ \\
\hline & Subtotal: & $28.8 \%$ & & & \\
\hline$\overline{81}$ & 71 & & 0 & & \\
\hline
\end{tabular}

Nota. Puntuación típica: 100 (media), 115 (+1 DS), 130 (+2 DS). P: Planificación; A: Atención; Si: Simultáneo. $23.6 \%$ de casos del total $28.8 \%$ de casos con algún proceso $>130$ es simultáneo $>130$ 
Figura 1. Perfil "N" a la izquierda y diferencia antre subescalas superior a 1 DS a la derecha. PLAN is planificación, SIM is simultáneo, AT is atención, and SEQ is secuencial

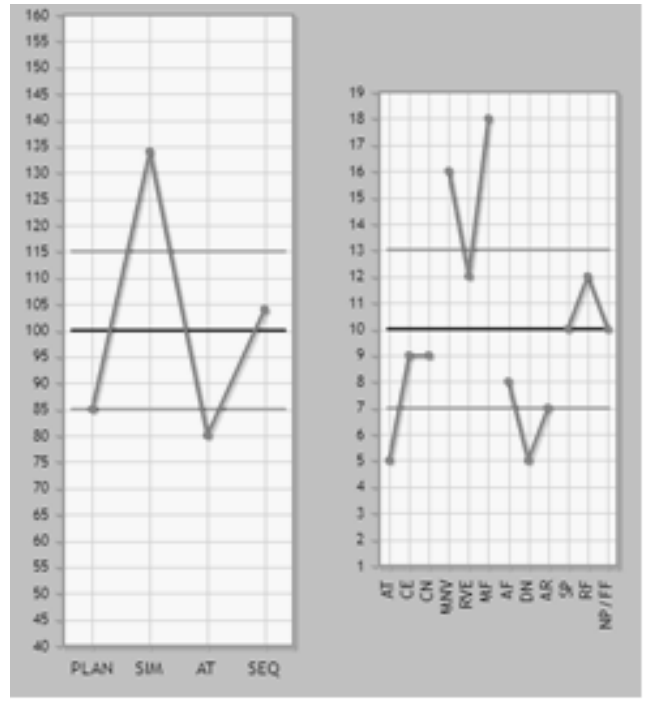

Finalmente, procedimos a investigar el número de perfiles "N" (Figura 1).

El perfil "N" consiste en un perfil que se dibuja cuando la planificación puntúa por debajo del simultáneo y, a su vez, la atención puntúa por debajo del simultáneo y del secuencial. La frecuencia de perfil " $N$ " fue significativamente más alta en la muestra de alta capacidad $(n=114)$ que en el grupo control de no alta capacidad $(n=100)\left(\chi^{2}=5.716 ; p=.016\right)$.

\section{DISCUSIÓN Y CONCLUSIONES}

Habíamos postulado que replicaríamos la estructura factorial del DN.CAS y así resultó ser (Tabla 1). Este estudio confirma por tanto que planificación y atención son procesamientos independientes con entidad propia, contrariamente a quienes consideran que ambos procesamientos son una misma entidad (Deng et al., 2011). Estos resultados avalan el modelo PASS (Das y Naglieri, 1997; Das, Naglieri, y Kirby, 1994; Das, Kar, y Parrilla, 1996; McCrea, 2009; Naglieri, 1999; Naglieri y Das, 1997; Swaiman, Ashwal, y Ferriero, 2006). Es cierto que planificación y atención muestran fuerte correlación entre sí (rs entre 0.49 y 0.60 ) (Tabla 2). Una fuerte correlación significa que ambas entidades son condiciones codependientes. Llamativamente, el WISC aparece como un factor sin relevante relación con los procesos PASS salvo en lo referente al simultáneo. El simultáneo, aunque correlaciona con planificación, atención y secuencial lo hace de forma débil o moderada (rs entre 0.20 y 0.49 ) lo que permite inferior que el simultaneo es el procesamiento más independiente de los demás. Varias pruebas del WISC aparecen 
asociadas al factor simultáneo. De manera complementaria, el análisis de correlación (Tabla 2) muestra fuerte correlación ( $r \geq 0.50)$ entre las pruebas WISC (rs entre 0.71 to 0.92 ), pero débil o moderada correlación entre WISC and DN-CAS lo que permite inferir que DN-CAS y WISC miden de manera diferente.

Lo anterior plantea algunas cuestiones. Primero de todo, la idoneidad de tanto WISC como DN-CAS para el diagnóstico de alta capacidad, particularmente, en casos de problemas de aprendizaje y disfunciones conductuales, casos definidos en la literatura como doblemente excepcionales. Postulamos que el hecho de que el DN-CAS mida planificación y el WISC no la mida supone una ventaja no despreciable a tener presente y una alternativa a los tests tradicionales que adolecen de debilidades incuestionables en este sentido (Nagleri, 1999). Es bien sabido que el CI verbal (Vocabulario, Aritmética y Semejanzas) ha resultado ser una medida de rendimiento y está influenciado por el conocimiento adquirido. El DN-CAS y el K-ABC, a diferencia del Wechsler, no tienen "ítems" altamente dependientes del conocimiento adquirido lo que elimina la posibilidad del efecto techo, es decir, que el conocimiento de la persona testada sea superior a los límites de la prueba (Naglieri y Kaufman, 2001). En cualquier caso, la teoría PASS de la inteligencia postula que mide procesamientos cognitivos a nivel neurológico central y no desenvolvimiento en una prueba determinada.

Se asume que, aunque necesario, un test de inteligencia no es suficiente para el diagnóstico. Sin embargo, lo estamos sugiriendo es que el DN-CAS ofrece una alternativa ventajosa. Un caso de alta capacidad puede pasar desapercibido sin una valoración de la planificación y viceversa. En cualquier caso, una valoración PASS podrá ayudar a definir con más precisión el perfil cognitivo del caso. Además, la planificación PASS se considera un indicador de creatividad (Kaufman, 2015). Creatividad e inteligencia tienden a correlacionar. Se considera que los tests tradicionales no son buenos indicadores de creatividad (Naglieri y Kaufman, 2001) en contraposición al papel importante de la planificación en la creatividad (Baker-Sennet, 1995). La planificación, a su vez, guarda estrecha relación con la metacognición o capacidad de evaluar nuestros pensamientos y conductas (Naglieri y Kaufmann, 2001).Ocurre, además, que la planificación está muy implicada en el razonamiento y es muy influenciable por la disfunción emocional siempre presente en casos de disfunción conductual y de disfunción de aprendizaje.

Dos estudios examinaron la relación entre los procesos PASS y la alta capacidad (Schofield y Ashman, 1987; Nagleri y Das, 1997). Schofield y Ashman hallaron que en caso de alta capacidad se da una mejor planificación y simultáneo, mientras que Naglieri y Das encontraron un mejor simultaneo y secuencial en estos casos. Nuestro estudio muestra que la alta capacidad está asociada a un mejor simultáneo.

Nuestro estudio investiga casos de alta capacidad con rendimiento académico disfuncional o disfunción conductual. Ambas situaciones pueden enmascarar la realidad de la alta capacidad. Personas cognitivamente brillantes no son académicamente brillantes o 
plantean problemas de conducta o las dos cosas a la vez. El profesorado no suele ver estos casos compatibles con alta capacidad. Un bajo rendimiento académico y una disfunción conductual pueden explicarse por una disfunción emocional. Se sabe que lo que se entiende como estrés puede incrementar la carga cognitiva, reducir la capacidad de la memoria de trabajo y ocasionar resultados en los tests por debajo de la capacidad real de la persona (Schmader y Johns, 2003).

El concepto de emoción ha sido interpretado en diferentes formas. En nuestra concepción, el procesamiento emocional del sentir configura la autoestima (confianza) y el autoconcepto (creencias personales). La confianza o seguridad y las creencias son inconscientemente memorizadas y neurológicamente codificadas tal que sentimientos negativos (dolorosos) son codificados como peligro o amenaza. Este concepto nos permite intervenir en la autoestima (seguridad personal) mediante la utilización de un procedimiento basada en la comunicación indirecta (hipnosis ericksoniana) siendo relevante la utilización de la metáfora (Pérez-Álvarez y Timoneda 2007; Pérez-Álvarez y Timoneda-Gallart, 2014; Pérez-Álvarez, Pérez-Serra, y Timoneda-Gallart, 2013). Este concepto implica que la disfunción académica o/y disfunción conductual son disfunciones enmascaradas, formas de expresión externa accesibles a la consciencia, pero solo son la consecuencia del estado de seguridad interior no accesible a la consciencia.

Nuestra amplísima experiencia con la utilización del DN-CAS nos ha permitido comprobar la asociación de un perfil que denominamos "N" (Figura 1) a disfunción emocional de manera que cuando revertimos el conflicto emocional la " $\mathrm{N}$ " desaparece porque la planificación se recupera alcanzando valores, al menos, similares al simultáneo. Hemos comprobado que la planificación es el procesamiento que responde más y mejor a una intervención emocional. El perfil " $\mathrm{N}$ " se asocia a diferencias entre las subescalas de planificación o/y simultaneo o/y atención o/y secuencial superiores a 1 DS (Pérez-Álvarez y Timoneda, 2007; Pérez-Álvarez y Timoneda-Gallart, 2014). De esta manera puede explicarse que los casos de nuestra muestra puntuaran en planificación menos que en el resto de procesamientos (Tablas 1 y 2).

En resumen, este estudio permite las siguientes conclusiones. Primero, el modelo factorial PASS (DN-CAS) de cuatro procesos aparece identificado en los casos de altas capacidades de la muestra. El WISC aparece como un factor muy diferente al PASS. El PASS se muestra consistente en culturas y lenguas diferentes. Un buen o excelente simultáneo aparece ligado a altas capacidades. Segundo, a diferencia del Wechsler, el DN-CAS, que no tiene "ítems" altamente dependientes del conocimiento adquirido y, por otra parte, mide la planificación, parece ofrecer ventajas para valorar altas capacidades, más si cabe en casos disfuncionales (casos doblemente excepcionales). Tercero, un perfil PASS particular, el perfil "N", aparece como un marcador o indicador de disfunción emocional.

El estudio presenta algunas limitaciones. Primero, la muestra no está seleccionada de una misma escuela lo que implica un no control del efecto derivado del 
ambiente y la instrucción. Segundo, no se usó una medida normalizada del rendimiento académico. Sería más convincente incluir medidas de este tipo en futuras investigaciones. Tercero, la generalización de los resultados está condicionada al tamaño de la muestra. En cualquier caso, se trataría de evidencia preliminar por la naturaleza exploratoria del estudio. Ello supone ampliar el conocimiento de la alta capacidad y una ayuda educacional. Estudios más amplios son deseables para confirmar o no estos resultados.

\section{REFERENCIAS}

Artola, T., Ancillo, I., Barraca, J., y Mosteiro, P. (2010). PIC-N. Prueba de Imaginación Creativa para Niños. [Creative Imagination Test for Children]. Madrid: TEA Ediciones.

Artola, T., Barraca, J., Martín-Azañedo, C., Mosteiro, P., Ancillo, I., y Poveda, B. (2008). PIC-J. Prueba de Imaginación Creativa para Jóvenes. [Creative Imagination Test for Young Children]. Madrid: TEA Ediciones.

Baker-Sennet, J. (1995). Improvisation, planning and the creative process. Infancia y Aprendizaje, 69, 111126.

Calero, M.D., García-Martín, M.B., y Robles, M.A. (2011). Learning Potential in High IQ Children: The Contribution of Dynamic Assessment to the Identification of Gifted Children. Learning and Individual Differences, 21(2), 176-181.

Corbalán, F.J., Martínez, F., Donolo, D.S., Alonso, C., Tejerina, M., y Limiñana, R.M. (2003). CREA. Inteligencia Creativa Una medida cognitiva de la creatividad. [Creative Intelligence. A cognitive measure of creativity]. Madrid. TEA Ediciones.

Craggs, J.G., Sanchez, J., Kibby, M.Y., Gilger, J.W., y Hynd, G.W. (2006). Brain morphology and neuropsychological profiles in a family displaying dyslexia and superior nonverbal intelligence. Cortex, 42(8), 1107-1118.

Dai, D.Y. (2005). Reductionism versus emergentism: a framework for understanding conceptions of giftednees. Roeper Review, 27, 144-151.

Das, J.P., y Naglieri, J.A. (1997). Cognitive Assessment System. Chicago, IL: Riverside Publishing.

Das, J.P., Naglieri, J.A., y Kirby, J.R. (1994). Assessment of cognitive processes. Needham \& Heights, MA: Allyn \& Bacon.

Das, J.P., Kar, R., y Parrilla, R.K. (1996). Cognitive planning. The psychological basis of intelligent behavior. London: Sage Publications.

Deng, C.P., Liu, M., Wei, W., Chan, R.C., y Das, J.P. (2011). Latent factor structure of the Das-Naglieri Cognitive Assessment System: A confirmatory factor analysis in a Chinese setting. Research in Developmental Disabilities, 32(5), 1988-1997.

Dole, S. (2000). The implications of the risk and resilience literature for gifted students with learning disabilities. Roeper Review, 23, 91-95.

Hernández, M.E, Speirs, K.L., Burney, V.H., y Cook, A.L. (2014). The Relationship of Cognitive and Executive Functioning With Achievement in Gifted Kindergarten Children. Gifted Child Quarterly, 58, 167-182

Kaufman, J.C. (2015). Why creativity isn't in IQ tests, why it matters, and why it won't change anytime soon probably. Journal of Intelligence, 3, 59-72.

McCrea, S.M. (2009). A review and empirical study of the composite scales of the Das-Naglieri: Cognitive Assessment System. Psychology Research and Behavior Management, 2, 59-79.

Naglieri, J.C. (1999). Essentials of CAS Assessment. New York: Wiley.

Naglieri, J.A., y Das, J.P. (1997). Das-Naglieri Cognitive Assessment System. Itasca, IL. Riverside.

Naglieri, J.A., y Kaufman, J.C. (2001). Understanding intelligence, giftedness and creativity using the PASS theory. Roeper Review, 23, 151-156. 
Nicpon, M.F., Allmon, A., Sieck, B., y Stinson, R. (2010). Empirical investigation of twice-exceptionality: where have we been and where are we going? Gifted Child Quarterly, 55, 3-17.

Pérez-Álvarez, F., y Timoneda, C. (2007). A Better Look at Intelligent Behavior. Hauppauge, NY: Nova Science Publishers, Inc.

Pérez-Álvarez, F., y Timoneda-Gallart, C. (2014). Intelligent behavior and neuroscience: What we knowand don't know-about how we think. En T.C. Papadopoulos, R.K. Parrila, y J.R. Kirby, Cognition, Intelligence, and Achievement: A Tribute to J.P. Das. (pp. 419-442). NY Elsevier Inc.

Pérez-Álvarez, F., Pérez-Serra, A., y Timoneda-Gallart, C. (2013). A better look at learning: how does the brain express the mind. Psychology, 4, 760-770.

Plucker, J.A. (2001). Looking back, looking around, looking forward: The impact of intelligence theories on gifted education. Roeper Review, 23, 124-125.

Reis, S.M., y McCoach, D.B. (2000). The underachievement of gifted students. What do we know and where do we go? Gifted Child Quaterly, 44, 158-170.

Robinson, S.M. (1999). Meeting the needs of students who are gifted and have learning disabilities. Journal of Learning Disabilities, 34, 195-204.

Schmader, T., y Johns, M. (2003). Converging evidence that stereotype threat reduces working memory capacity. Journal of Personality and Social Psychology, 85(3), 440-452.

Schofield, N.J., y Ashman, A.F. (1987). The cognitive processing of gifted, high average and low average ability students. British Journal of Educational Psychology, 57(1), 9-20.

Shore, B.M. (2000). Metacognition and flexibility: Qualitative differences in how gifted children think. In R.C. Friedman, \& B.M. Shore (Eds.), Talents unfolding: Cognition and development (pp. 17187). Washington, DC: American Psychological Association.

Steiner, H.H., y Carr, M. (2003). Cognitive development in gifted children: toward a more precise understanding of emergent differences in intelligence. Educational Psychology Review, 15, 215246.

Steiner, H.H. (2006). A micro genetic analysis of strategic variability in gifted and average-ability children. Gifted Child Quarterly, 50, 62-74.

Sternberg, R.J., Jarvin, L., y Grigorenko, E.L. (2011). Exploration in giftedness. New York: Cambridge. University Press.

Swaiman, K.F., Ashwal, S., and Ferriero, D.M. (2006). Pediatric Neurology. Principles \& Practice. 4th ed. Philadelphia, PA: Mosby Inc.

Yuste, C., Martínez, R., y Galve, J.L. (2002). Batería de aptitudes diferenciales y generales. [Differential and general skills battery]. BADyG-BADyG-M. CEPE. Madrid.

Webb, J.T. (2001). Mis-diagnosis and dual diagnosis of Gifted children: Gifted and LD, ADHD, OCD, oppositional defiant disorder. En N. Hafenstein y F. Rayney, (Eds.), Perspectives in gifted education: Twice excepcional children (pp. 23-31). Denver: Ricks Center for Gifted Children, University of Denver.

Wechsler, D. (2003). WISC-IV technical and interpretive manual. San Antonio, TX: Psychological Corporation.

Weinfeld, R., Barnes-Robinson, L., Jeweler, S., y Shevitz, B. (2002). Academic programs for gifted and talented/learning disabled students. Roeper Review, 24, 226-233.

Recibido: 21 de abril de 2019

Recepción Modificaciones: 29 de abril de 2019

Aceptado: 5 de mayo de 2019 